\title{
Transitions in microbial communities \\ along two sediment corescollected from the landward walls of the New Britain trench
}

\author{
LI WANG, RULONG LIU AND JIASONG FANG \\ Shanghai Ocean University \\ Presenting Author: 1-wang@shou.edu.cn
}

The steep landward walls with a funneling shape favor the accumulation of organic carbon for the trench area. The kind of allochthonous inputs to trenches may be pre-aged as a consequence of their pre-depositional histories (storage in intermediate reservoirs on the continent and/or the margin). Microbial metabolism in the trench walls may affect the components of input carbon for the trench. However, our understanding about the microbiology of the trench walls is limited. In this study, we collected a $70 \mathrm{~cm}$ gravity core $(4524 \mathrm{~m}$ depth) and a $30 \mathrm{~cm}$ box core (4130 $\mathrm{m}$ depth) from the western and eastern landward walls of the New Britain Trench (NBT). Diversity and composition of microbial communities were investigated at high resolution in $2 \mathrm{~cm}$, total 29 sediment samples. Obvious vertical transitions from the aerobic to anaerobic but distinct dominant anaerobic microbes were observed in two cores. In the steeper eastern trench wall, anaerobic fermenters, such as Anaerolineales and Alphaproteobacteria, were dominant, but the western contained abundant Dehalococcoidia, and Gammaproteobacteria, such as Alteromonadales and Oceanospirillales. Combined with the results of higher bacteria abundance and probable shallower aerobic active zone in the eastern core, such patterns of species composition might be related with richer input of labile allochthonous terrestrial organic matter by mass wasting processes in the steeper eastern wall. Our analysis also revealed highly diversified Dehalococcoidia including some new phylotypes, extending the current understandings on Dehalococcoidia diversity. This study fills the gap of benthic microbial study in the landward wall of NBT, and highlights the effect of geomorphology (especially the slant of continental slope) on microbial community along the trench wall. 